\title{
Application of contrast-enhanced T1-weighted MRI-based 3D reconstruction of the dural tail sign in meningioma resection
}

\author{
Binsheng You, MM, ${ }^{1}$ Yanhao Cheng, MM, ${ }^{2}$ Jian Zhang, MD, ${ }^{2}$ Qimin Song, MM, ${ }^{2}$ Chao Dai, BM, ${ }^{2}$ \\ Xueyuan Heng, BM, ${ }^{2}$ and Chang Fei, BM ${ }^{2}$ \\ 'Department of Neurosurgery, Xuzhou Medical College, Xuzhou, Jiangsu Province; and ²Department of Neurosurgery, Linyi
People's Hospital, Linyi, Shandong Province, China
}

\begin{abstract}
OBJECTIVE The goal of this study was to investigate the significance of contrast-enhanced T1-weighted (T1W) MRIbased 3D reconstruction of dural tail sign (DTS) in meningioma resection.

METHODS Between May 2013 and August 2014, 18 cases of convexity and parasagittal meningiomas showing DTS on contrast-enhanced T1W MRI were selected. Contrast-enhanced T1W MRI-based 3D reconstruction of DTS was conducted before surgical treatment. The vertical and anteroposterior diameters of DTS on the contrast-enhanced T1W MR images and 3D reconstruction images were measured and compared. Surgical incisions were designed by referring to the 3D reconstruction and MR images, and then the efficiency of the 2 methods was evaluated with assistance of neuronavigation.
\end{abstract}

RESULTS Three-dimensional reconstruction of DTS can reveal its overall picture. In most cases, the DTS around the tumor is uneven, whereas the DTS around the dural vessels presents longer extensions. There was no significant difference $(p>0.05)$ between the vertical and anteroposterior diameters of DTS measured on the contrast-enhanced T1W MR and 3D reconstruction images. The 3D images of DTS were more intuitive, and the overall picture of DTS could be revealed in 1 image, which made it easier to design the incision than by using the MR images. Meanwhile, assessment showed that the incisions designed using 3D images were more accurate than those designed using MR images (ridit analysis by $S A S, F=7.95 ; p=0.008$ ). Pathological examination showed that 34 dural specimens (except 2 specimens from 1 tumor) displayed tumor invasion. The distance of tumor cell invasion was 1.0-21.6 mm (5.4 $\pm 4.41 \mathrm{~mm}$ [mean \pm SD]). Tumor cell invasion was not observed at the dural resection margin in all 36 specimens.

CONCLUSIONS Contrast-enhanced T1W MRI-based 3D reconstruction can intuitively and accurately reveal the size and shape of DTS, and thus provides guidance for designing meningioma incisions.

http://thejns.org/doi/abs/10.3171/2015.5.JNS142510

KEY WORDS meningioma; dural tail sign; 3-dimensional reconstruction; neuronavigation; oncology

$I^{1}$ N 1989, Wilms et al..$^{14}$ described thickening of the dura mater adjacent to a meningioma on contrast-enhanced T1-weighted (T1W) MRI, which was called the "dural tail sign (DTS)," "dural thickening," "flare," or "meningeal sign." In 1990, Goldsher et al. ' developed the following criteria for the DTS: 1) presence at least 2 consecutive sections through the tumor at the same site and in more than 1 plane, 2) greatest thickness adjacent to the tumor and tapering away from it, and 3) enhancement greater than that of the tumor mass itself.

There is no consistent information on the pathophysi- ological mechanism of the DTS. Wilms et al. ${ }^{14}$ found that the thickened dura is accompanied by an invasion of tumor cells and, for the first time, considered the DTS to be a marker for tumor invasion. In contrast to the study by Wilms and colleagues, Tokumaru et al. ${ }^{13}$ believed that the DTS was mainly caused by the proliferation of connective tissue and dilation of blood vessels. Although in some patients there was found to be dural invasion by tumor cells, the invasion was confined to only within $1 \mathrm{~mm}$. RokniYazdi et al. ${ }^{10}$ found that in $34.1 \%$ of cases, the DTS was invaded by tumor cells. Kawahara et al. ${ }^{5}$ conducted pathol- 
ogy studies on the attachment point of meningioma and its adjacent dura mater. They suggested that tumor cells first invade vessels, packing them at this point. Then, the adjacent dura mater becomes congested with loose connective-tissue proliferation, hypervascularity, and dilation of vessels. As a result, it is enhanced markedly, giving the DTS.

Although the mechanism by which the DTS forms in meningiomas remains controversial, it has been confirmed that at least parts of the DTS were invaded by tumor cells. ${ }^{10}$ Because it is very difficult to accurately identify before surgery whether the DTS is due to the infiltration of tumor cells or reactive blood vessel hyperplasia, most scholars believe that the DTS should be removed to reduce tumor recurrence. $8,9,11,12$

Resection of the tumor and its attached dura is the main treatment for meningioma. It is generally believed that the postoperative recurrence of meningiomas is closely related to resection grade., ${ }^{411,15}$ It has been reported that the recurrence rate at 5 years was $9 \%$ for Simpson Grade I excision, $19 \%$ for Simpson Grade II excision, and 29\% for Simpson Grade III excision. ${ }^{15}$

Because parts of the DTS in meningiomas are very long and/or have irregular edges, the incisions might not be large enough to include the entire DTS. This is likely to cause incomplete resection of the DTS and induce tumor recurrence. We conducted contrast-enhanced T1W MRI-based 3D reconstruction of the DTS on 18 cases of convexity and parasagittal meningiomas between May 2013 and August 2014 in the Department of Neurosurgery at Linyi People's Hospital. The results revealed that 3D reconstruction can intuitively and accurately reveal the DTS, aid in the design of the incision, and increase the complete resection rate.

\section{Methods \\ Clinical Data}

We received approval for this study from the Linyi People's Hospital ethics committee and obtained informed consent from patients. Between May 2013 and August 2014, 18 cases of convexity and parasagittal meningiomas with the DTS, as shown on contrast-enhanced T1W MRI, were selected. After obtaining the consent of patients and their families, preoperative 3D reconstruction of the DTS was conducted to guide design of the surgical incision and plan the resection of dura mater. Of the 18 patients, there were 12 cases with convexity meningiomas and 6 cases with parasagittal meningiomas. The tumor diameters ranged from 1.2 to $5.3 \mathrm{~cm}(3.4 \pm 0.76 \mathrm{~cm}[$ mean $\pm \mathrm{SD}])$. There were 13 women and 5 men who ranged in age from 26 to 62 years (mean 52.3 years).

\section{Three-Dimensional Reconstruction of DTS}

Four to eight positioning markers were affixed to the patient's head to prepare for the subsequent neuronavigation. After injection of Gd-DTPA enhancer, the Siemens Verio 3-T superconductive MR unit was used for the 3D fast low-angle shot T1W multiplanar image reconstruction serial axial scans at a thickness of $1.5 \mathrm{~mm}$.

The data were then imported into the syngo MR B17
(Siemens) that came with the Siemens Verio workstation and were opened with a 3D task card. Three-dimensional reconstruction was conducted using the surface-shaded display functions. ${ }^{1}$ The syngo 3D cutting tools were used to virtually strip the scalp and skull. Then, the maximum intensity projection and volume rendering for tissues were used to reveal the dural structures.

\section{Measurement of DTS}

The vertical diameters of the DTS were measured in the contrast-enhanced coronal T1W MR images, and the anteroposterior diameters were measured in the contrastenhanced axial T1W MR images of the convexity meningiomas and the sagittal scan images of the parasagittal meningiomas. The vertical and anteroposterior diameters were also measured in the $3 \mathrm{D}$ reconstruction images of DTS. All measurements were completed independently by 1 senior neurosurgeon.

\section{Incision Design and Verification With Neuronavigation}

The images were imported into an electromagnetic neuronavigation workstation. The patient received general anesthesia, a head frame was installed, and a magnetic launcher was connected. A navigation probe was then used to register each marker, and images were automatically displayed on the system with a control error $<2.0$ $\mathrm{mm}$.

A horseshoe incision was adopted for all patients. To fully reveal the DTS, the incision should be $2 \mathrm{~cm}$ larger than both the vertical and anteroposterior diameters (e.g., if the DTS is $4 \times 5 \mathrm{~cm}$, the incision should be $6 \times 7 \mathrm{~cm}$ ), which would ideally reveal a normal dura $1 \mathrm{~cm}$ around the DTS (Fig. 1A). The surgical incision was designed separately by 2 randomly selected senior neurosurgeons. One neurosurgeon designed the incision based on the 3D reconstruction of the DTS. In this case, the borders of the DTS and the tumor were identified with neuronavigation, and the pros and cons of the incision were evaluated. As a control group, after eliminating marks of incision, the second neurosurgeon designed the incision based on a contrast-enhanced T1W MR image. Again, the borders of the DTS and the tumor were identified with neuronavigation, and the incision was evaluated (Fig. 1).

In evaluating the incision, cases in which the distance from the DTS boundary to the incision was $\geq 0.5 \mathrm{~cm}$ were considered excellent (Fig. 1A); those $<0.5 \mathrm{~cm}$ were considered good (Fig. 1B), cases with a DTS that extended beyond the incision were considered fair (Fig. 1C), and cases in which the incision did not completely encompass the tumor were considered poor (Fig. 1D).

\section{Results}

\section{Three-Dimensional Reconstruction of the DTS}

Preoperative 3D reconstruction of the DTS in 18 patients successfully revealed the overall picture of the objects. The 3D images were very distinct and more intuitive than cross-sectional images. In most cases, the DTS around the tumor was uneven, and parts of it around the dural vessels presented longer extensions. In only a few cases did the DTS exhibit relatively regular edges. Some 
B. You et al.
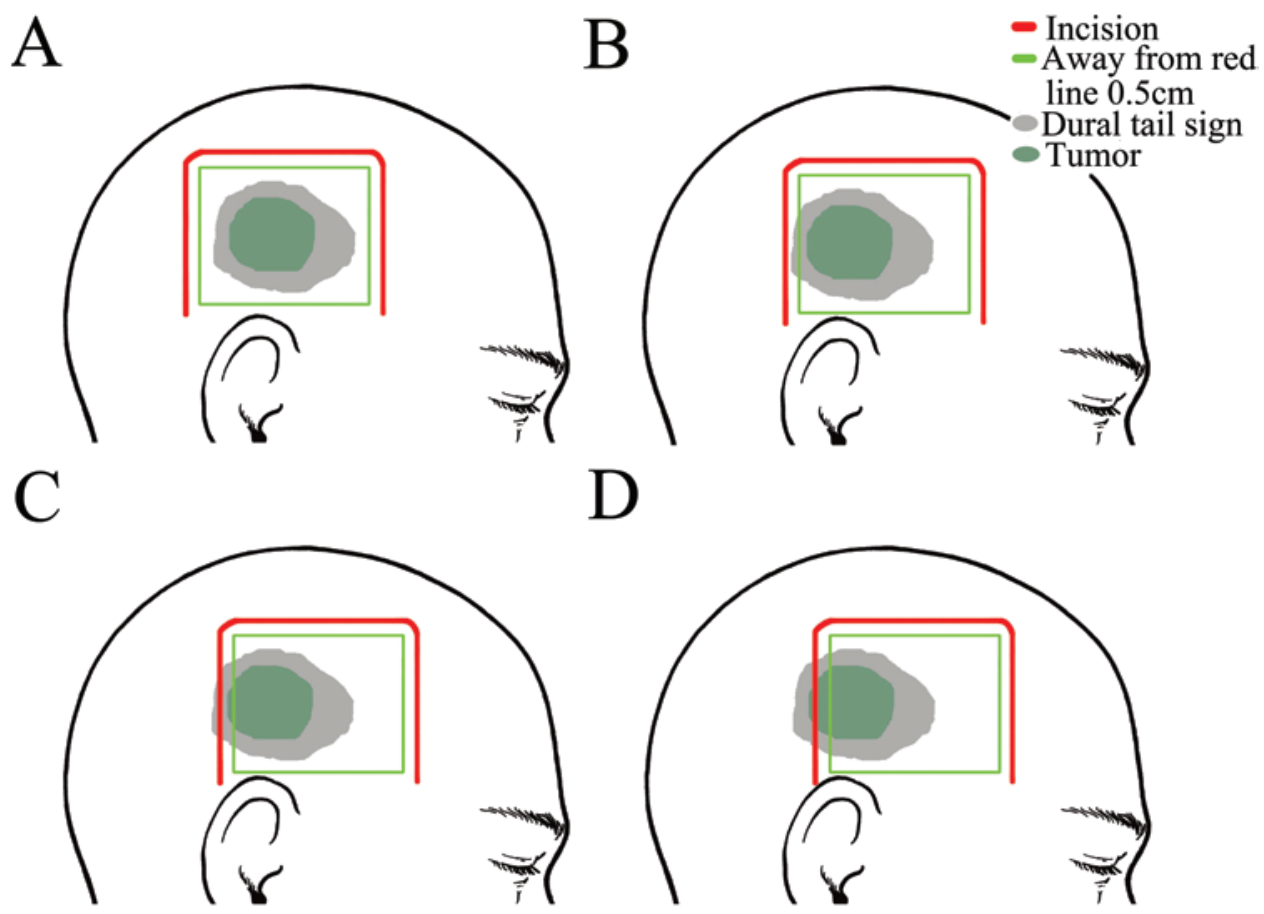

FIG. 1. The distance from the green line to the predicted incision (red line) is $0.5 \mathrm{~cm}$. Incision evaluation was rated as follows. A: Excellent, distance from dural tail boundary to incision $\geq 0.5 \mathrm{~cm}$. B: Good, distance from dural tail boundary to incision $<0.5$ $\mathrm{cm}$. C: Fair, dural tail extends beyond the incision. D: Poor, tumor not completely encompassed by the incision.

small multiple meningiomas, which were often ignored on MRI, displayed more clearly on $3 \mathrm{D}$ reconstruction of the DTS. Moreover, 3D reconstruction of the DTS could illustrate the blood supply, venous return, and its relationship with the neighboring sinus (Fig. 2).

\section{Comparison of DTS Revealed by 3D Reconstruction Versus Contrast-Enhanced T1W MRI}

The mean vertical diameter of the DTS in 18 cases measured in contrast-enhanced T1W MR images was $4.55 \mathrm{~cm}$, whereas that in 3D images was $4.58 \mathrm{~cm}$; the
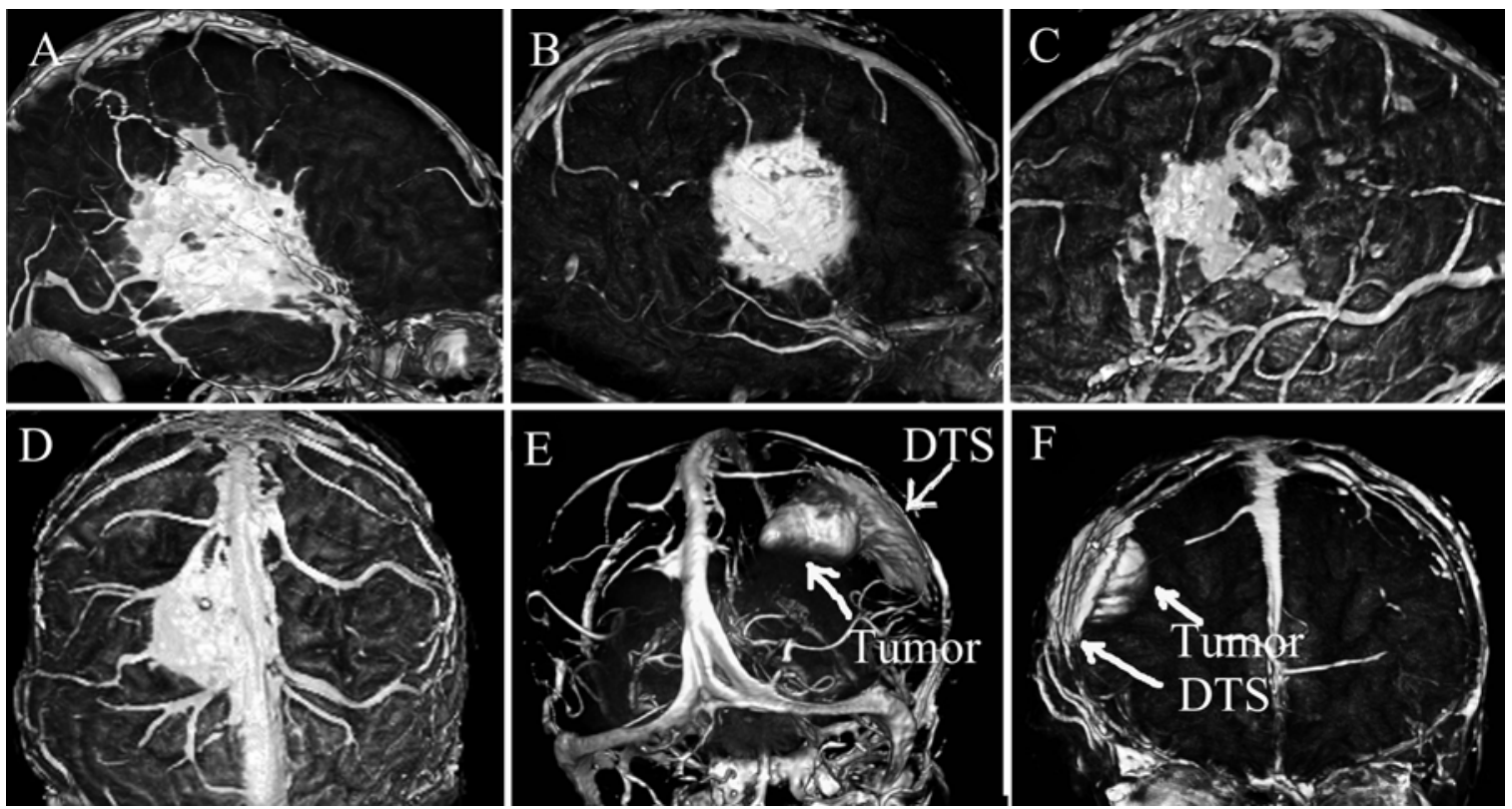

FIG. 2. Overall pictures of the DTS obtained from contrast-enhanced T1W MRI-based 3D reconstruction. A: In most cases, the DTS around the tumor was uneven. B: In a few cases, the DTS exhibited relatively regular edges. C: Multiple meningiomas. D: Invasion of the neighboring sinus. E and F: DTS and tumor (arrows). 
TABLE 1. Vertical and anteroposterior diameters of DTS measured in contrast-enhanced T1W MR images and 3D reconstruction images (18 cases)

\begin{tabular}{lcc}
\hline \multicolumn{1}{c}{ Type of Image } & $\begin{array}{c}\text { Vertical Diameter, } \\
\text { Mean } \pm \text { SD }\end{array}$ & $\begin{array}{c}\text { Anteroposterior } \\
\text { Diameter, } \\
\text { Mean } \pm \text { SD }\end{array}$ \\
\hline Contrast-enhanced T1W MR & $4.55 \pm 1.26 \mathrm{~cm}$ & $4.73 \pm 1.21 \mathrm{~cm}$ \\
\hline 3D reconstruction & $4.58 \pm 1.27 \mathrm{~cm}$ & $4.78 \pm 1.23 \mathrm{~cm}$ \\
\hline$p$ Value (t-test) & 0.930 & 0.915 \\
\hline
\end{tabular}

difference between these 2 groups was not significant (ttest using SAS [version 9.2; SAS Institute], $\mathrm{t}=-0.09 ; \mathrm{p}$ $=0.930$ ). The mean anteroposterior diameter measured in the contrast-enhanced T1W MR images and in the 3D images were $4.73 \mathrm{~cm}$ and $4.78 \mathrm{~cm}$, respectively; the difference was not statistically significant (t-test using SAS, $\mathrm{t}=-0.11 ; \mathrm{p}=0.915$ ) (Table 1). These results suggest that the size of the DTS revealed by $3 \mathrm{D}$ reconstruction versus contrast-enhanced T1W MRI was consistent (Fig. 3).

\section{Guidance by MR Images Versus 3D Images of DTS in Incision Design}

It is difficult to design an incision based on the MR image; axial, coronal, and sagittal planes must be consulted. The assessment of the incision showed that there were 2 excellent cases and 8 good cases. In 5 cases, DTS appeared to extend beyond the incision, and in 3 cases, the incision did not completely encompass the tumor. However, the 3D images of DTS were more intuitive, and the overall picture of the DTS could be revealed in 1 image, which made it easier to design the incision (Fig. 4). The evaluation results showed that there were 7 excellent cases and 9 good cases, and only 2 cases presented a DTS extending beyond the incision (Table 2). These results indicated that the incisions designed using the 3D images were more accurate than those designed using the MR images (ridit analysis using SAS, $F=7.95 ; p=0.008$ ).

\section{Surgery}

After using neuronavigation to adjust the incision, the craniotomy was performed and the dural incision was made along the periphery of the DTS. Neuronavigation guidance was used to remove the tumor as well as the DTS. Among the 18 cases of meningiomas, complete resection of the tumor and DTS was accomplished in 16 cases (including 1 patient who underwent partial resection of the sagittal sinus wall and repair using an artificial dura because the sinus was invaded by the tumor), and 2 patients underwent dural coagulation because the DTS extended to the surface of the sagittal sinus wall.

\section{Histopathological Examination}

Gross morphological observation of the DTS indicated that $14(77.8 \%)$ cases were obviously accompanied by an invasion of tumor (Figs. 4E and 5). Due to the unequal DTS around the tumor, we chose the longest and shortest parts for pathological examination in this study. The length of 36 embedded dural specimens ranged from 3.8 to $26.5 \mathrm{~mm}$ (mean $10.1 \pm 5.1 \mathrm{~mm}$ ). Thirty-four dural specimens displayed tumor invasion, vascular proliferation, and dilation (Fig. 6), and the other 2 specimens (from the same tumor) demonstrated vessel proliferation without tumor invasion. The distance of tumor cell invasion was 1.0-21.6 $\mathrm{mm}$ (mean $5.4 \pm 4.41 \mathrm{~mm}$, measuring on tissue sections).
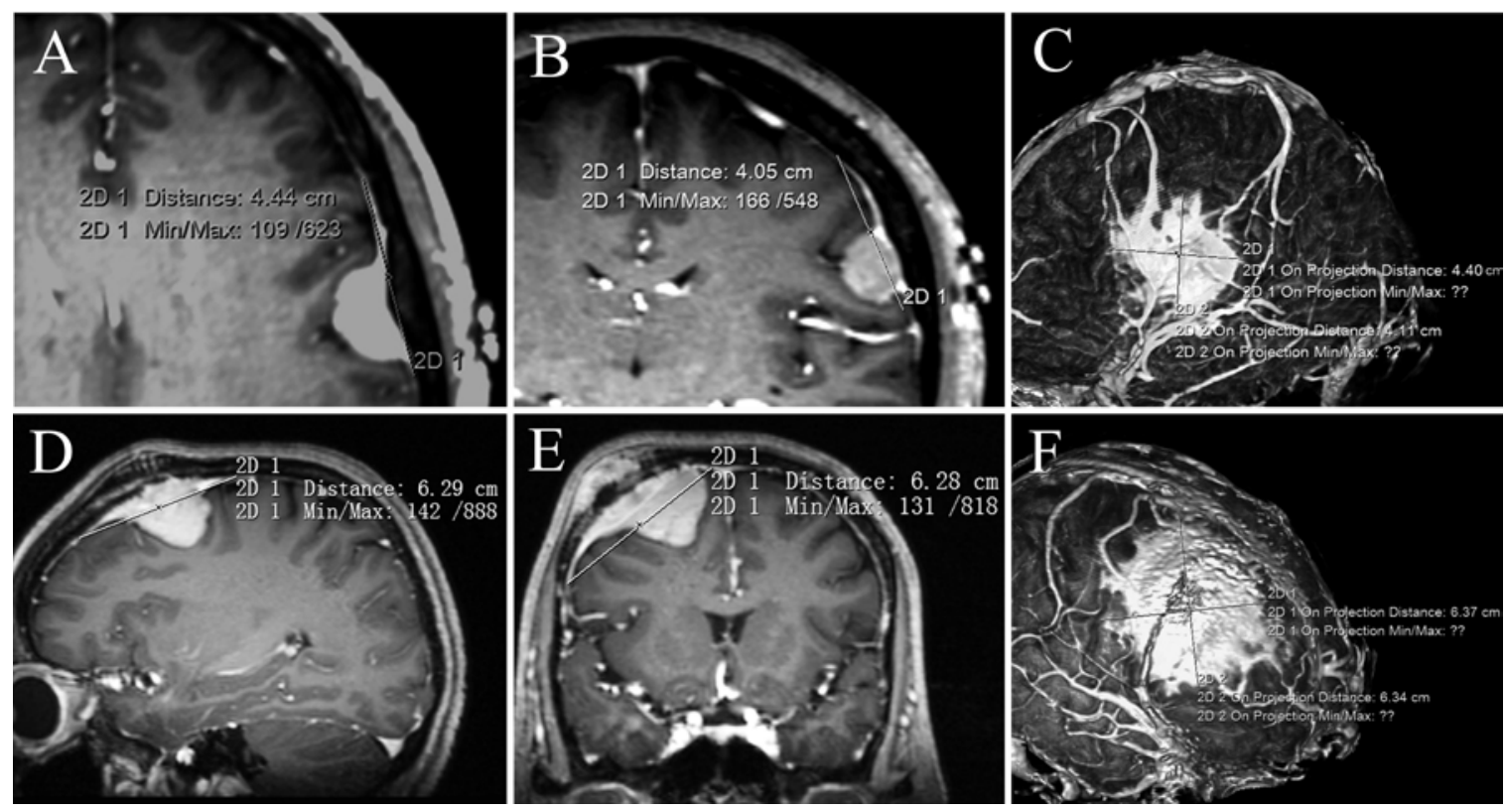

FIG. 3. Convexity meningiomas. Measuring anteroposterior diameter of the DTS on axial image (A) and vertical diameter on coronal image (B). Measuring anteroposterior and vertical diameters of the DTS on the 3D image (C). Parasagittal meningiomas. Measuring anteroposterior diameter of the DTS on axial image (D) and vertical diameter on coronal image (E). Measuring anteroposterior and vertical diameters of the DTS on the 3D image (F). 

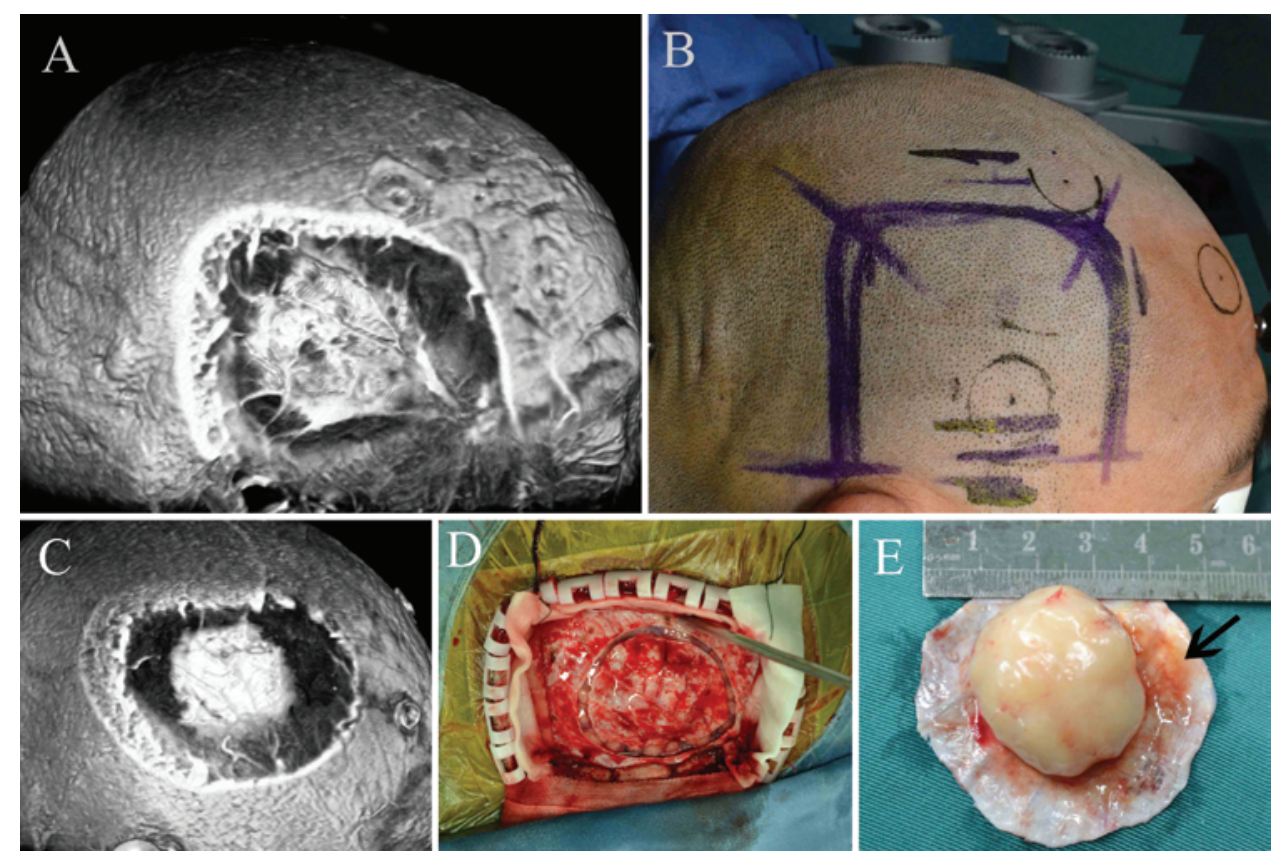

FIG. 4. A: 3D reconstruction image of the DTS of temporal meningioma. B: Incision based on 3D reconstruction image of the DTS. C: 3D reconstruction image of the DTS of frontotemporal meningioma. D: The dural incision along the periphery of the DTS. E: DTS and tumor. Tumor cell invasion almost reached the outer edge of the DTS (arrow). Figure is available in color online only.

In a few cases, tumor cell invasion almost reached the outer edge of the DTS (Fig. 4E). Tumor cell invasion was not observed at the dural resection margin in all 36 specimens.

\section{Discussion}

Contrast-enhanced T1W MRI-based 3D reconstruction is a new noninvasive imaging technique that has been developed in recent years. It can clearly reveal the structure of the brain surface and present the relationship between lesions and their surrounding normal nerves and vessels. With preoperative $3 \mathrm{D}$ reconstruction of brain tissues, we can more fully understand the meningioma blood supply, venous return, and invasion of the neighboring sinus and its relationship with the surrounding normal brain tissues. This provides guidance for the design of a surgical incision and protection of important structures. , $^{13,6,7,16}$

To our knowledge, no published reports have focused on 3D reconstruction of the DTS. In this study, contrastenhanced T1W MRI was used because it presented very

TABLE 2. Comparison of incisions designed by contrast-enhanced T1W MR images versus 3D reconstruction images of DTS*

\begin{tabular}{lccccc}
\hline \multirow{2}{*}{ Type of Evaluation } & \multicolumn{5}{c}{ Rating } \\
\cline { 2 - 6 } & Excellent & Good & Fair & Poor & Total \\
\hline $\begin{array}{l}\text { Referred to enhanced T1W } \\
\text { MR images }\end{array}$ & 2 & 8 & 5 & 3 & 18 \\
\hline Referred to 3D images & 7 & 9 & 2 & 0 & 18 \\
\hline
\end{tabular}

* $F=7.95 ; p=0.008$; ridit analysis using SAS. clear images of the DTS. Continuous axial scanning was performed after injection of a contrast agent, and 3D reconstruction of the DTS was developed. The results showed that the overall picture of the DTS can be revealed in 1 image. The image was very clear and intuitive, revealing that in most cases the DTS around the tumor was uneven and usually extended farther around the dural vessels, which is sometimes very difficult to observe on sagittal, coronal, and axial planes on enhanced MRI (Figs. 2A and $\mathrm{E}, 3 \mathrm{C}$ and $\mathrm{F}$ ). Moreover, 3D reconstruction of the DTS could present multiple meningiomas more clearly, particu-

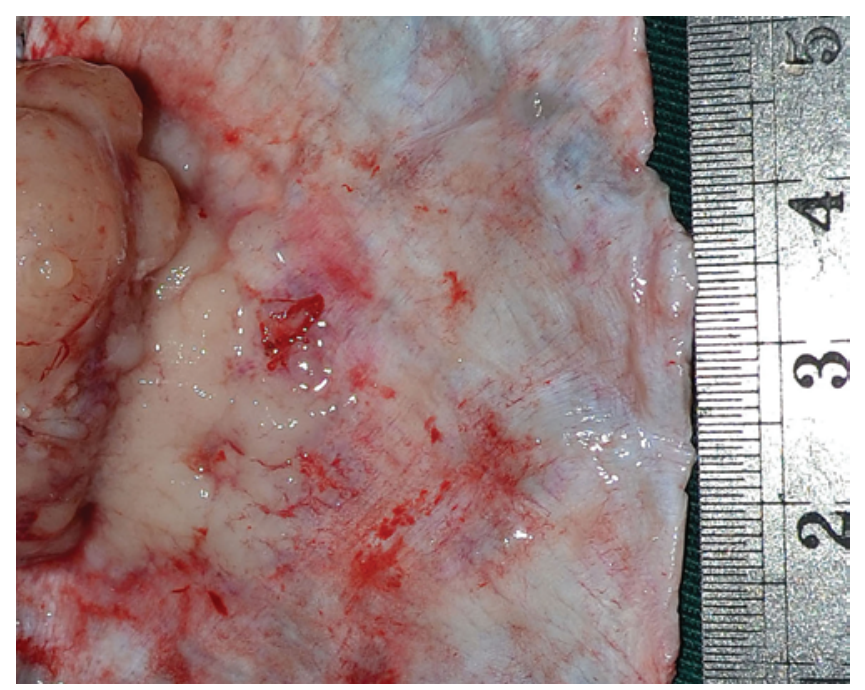

FIG. 5. Gross morphological observation revealed a DTS with tumor invasion and vascular proliferation. Figure is available in color online only. 


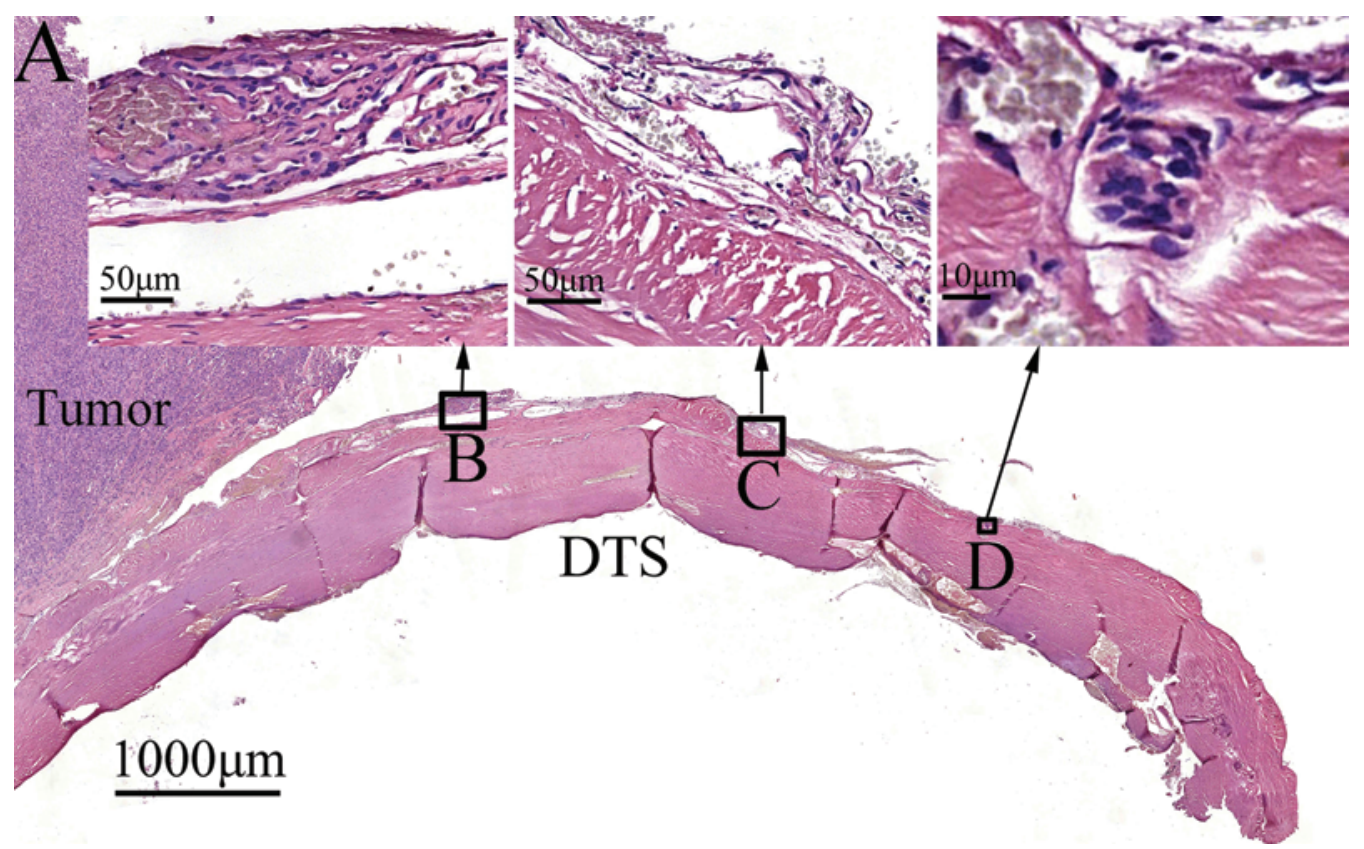

FIG. 6. Pathological examination. A: Tumor and DTS. B: DTS with tumor invasion and vascular proliferation on the surface of the dura. C: DTS with vascular proliferation and without tumor invasion. D: DTS with tumor invasion and vascular proliferation. H \& E. Original magnification $\times 10(A), \times 100(B$ and $C)$, and $\times 200(D)$. Figure is available in color online only.

larly the small lesions around the main tumor which were often ignored on MRI (Fig. 2C).

Can 3D reconstruction display DTS accurately and fully? This study showed that there was no significant difference between the vertical and anteroposterior diameters obtained by $3 \mathrm{D}$ reconstruction and the contrast-enhanced T1W MRI. This suggested that the size of DTS obtained by $3 \mathrm{D}$ reconstruction and by contrast-enhanced T1W MRI were consistent with each other.

The pathophysiological mechanism of the DTS is still controversial. Some authors believe that the DTS is often accompanied by tumor cell invasion..$^{10,14}$ Others argue that the DTS is mainly caused by the proliferation of connective tissue and dilation of blood vessels. ${ }^{5,13}$ According to the present study, gross morphological observation of the DTS indicated that $14(77.8 \%)$ cases were obviously accompanied by an invasion of tumor cells, whereas 34 (94.4\%) dural specimens displayed tumor invasion, vascular proliferation, and dilation under microscope. The longest invasion distance reached $21.6 \mathrm{~mm}$ (Fig. 5). The actual distance of tumor invasion might be more than that because the tumor and DTS shrank while undergoing excision, formalin fixation, and paraffin embedding. It is important to note that tumor cell invasion almost reached the outer edge of the DTS in a few cases (Fig. 4E). Therefore, the DTS should be resected with the tumor to decrease recurrence rates of meningiomas.

Planning and implementation of meningioma surgery rely mainly on guidance of sagittal, coronal, and axial planes on CT and MRI, which are unlikely to provide an intuitive and overall picture to neurosurgeons. This study showed that it is difficult to design an incision based on the MR image alone, because there is a high incidence of incomplete coverage of the DTS and tumor. Therefore, re- liance solely on the MR image is likely to result in DTS residues if no neuronavigation guidance is used.

\section{Conclusions}

Compared with MR images, 3D reconstruction images are more intuitive. Also, the overall picture of a DTS can be revealed in 1 image, which makes it easier to design an incision. After performing the craniotomy, the dural incision can be made along the periphery of the DTS based on the $3 \mathrm{D}$ images, and ultimately, the tumor and invaded dura can be completely removed. Thus, 3D images of DTS have a high application value and are especially suitable for hospitals that do not have a neuronavigation system.

\section{References}

1. Aydin K, Cokluk C, Kuruoglu E, Gelmez S, Diren B, Rakunt $\mathrm{C}$, et al: Using the magnetic resonance three-dimensional volume rendering for tissues technique in the planning of craniotomy flaps with linear scalp incision. Minim Invasive Neurosurg 49:189-193, 2006

2. Goldsher D, Litt AW, Pinto RS, Bannon KR, Kricheff II: Dural "tail" associated with meningiomas on Gd-DTPAenhanced MR images: characteristics, differential diagnostic value, and possible implications for treatment. Radiology 176:447-450, 1990

3. Jung HW, Chang KH, Choi DS, Han MH, Han MC: Contrastenhanced MR angiography for the diagnosis of intracranial vascular disease: optimal dose of gadopentetate dimeglumine. AJR Am J Roentgenol 165:1251-1255, 1995

4. Kalala JP, Caemaert J, De Ridder L: Primary resected meningiomas: relapses and proliferation markers. In Vivo 18:411416, 2004

5. Kawahara Y, Niiro M, Yokoyama S, Kuratsu J: Dural congestion accompanying meningioma invasion into vessels: the dural tail sign. Neuroradiology 43:462-465, 2001 
6. Kirchhof K, Welzel T, Jansen O, Sartor K: More reliable noninvasive visualization of the cerebral veins and dural sinuses: comparison of three MR angiographic techniques. Radiology 224:804-810, 2002

7. Liang L, Korogi Y, Sugahara T, Onomichi M, Shigematsu Y, Yang D, et al: Evaluation of the intracranial dural sinuses with a 3D contrast-enhanced MP-RAGE sequence: prospective comparison with 2D-TOF MR venography and digital subtraction angiography. AJNR Am J Neuroradiol 22:481492, 2001

8. Nakamura M, Tsuji O, Fujiyoshi K, Hosogane N, Watanabe $\mathrm{K}$, Tsuji T, et al: Long-term surgical outcomes of spinal meningiomas. Spine (Phila Pa 1976) 37:E617-E623, 2012

9. Qi ST, Liu Y, Pan J, Chotai S, Fang LX: A radiopathological classification of dural tail sign of meningiomas. J Neurosurg 117:645-653, 2012

10. Rokni-Yazdi H, Azmoudeh Ardalan F, Asadzandi Z, Sotoudeh H, Shakiba M, Adibi A, et al: Pathologic significance of the "dural tail sign". Eur J Radiol 70:10-16, 2009

11. Simpson D: The recurrence of intracranial meningiomas after surgical treatment. J Neurol Neurosurg Psychiatry 20:22-39, 1957

12. Sotoudeh H, Yazdi HR: A review on dural tail sign. World J Radiol 2:188-192, 2010

13. Tokumaru A, O'uchi T, Eguchi T, Kawamoto S, Kokubo T, Suzuki M, et al: Prominent meningeal enhancement adjacent to meningioma on Gd-DTPA-enhanced MR images: histopathologic correlation. Radiology 175:431-433, 1990

14. Wilms G, Lammens M, Marchal G, Van Calenbergh F, Plets C, Van Fraeyenhoven L, et al: Thickening of dura surrounding meningiomas: MR features. J Comput Assist Tomogr 13:763-768, 1989
15. Whittle IR, Smith C, Navoo P, Collie D: Meningiomas. Lancet 363:1535-1543, 2004

16. Yang JJ, Hill MD, Morrish WF, Hudon ME, Barber PA, Demchuk AM, et al: Comparison of pre- and postcontrast 3D time-of-flight MR angiography for the evaluation of distal intracranial branch occlusions in acute ischemic stroke. AJNR Am J Neuroradiol 23:557-567, 2002

\section{Disclosures}

The authors report no conflict of interest concerning the materials or methods used in this study or the findings specified in this paper.

\section{Author Contributions}

Conception and design: Zhang, You. Acquisition of data: You, Cheng, Song, Dai, Heng, Fei. Analysis and interpretation of data: Zhang, You, Cheng, Song, Dai. Drafting the article: all authors. Critically revising the article: Zhang, You, Cheng. Reviewed submitted version of manuscript: Zhang, You, Cheng, Song, Dai. Approved the final version of the manuscript on behalf of all authors: Zhang. Statistical analysis: Zhang, You, Cheng, Song. Study supervision: Zhang, Heng, Fei.

\section{Correspondence}

Jian Zhang, Department of Neurosurgery, The People's Hospital of Linyi City, 49 Yizhou Rd., Linyi City, Shandong Province 276000, China. email: honest20699@126.com. 